\title{
Analysis of global manufacturing virtual networks in the aeronautical industry
}

\author{
Carlos Rodríguez Monroy, José Ramón Vilana Arto* \\ Departamento de Ingeniería de Organización, Administración de Empresas y Estadística, E.T.S.I.I., Universidad Politécnica de Madrid, Madrid, Spain
}

\begin{abstract}
A B S T R A C T
The evolution of organizations that work in multinational environments has considerably altered their production strategies. One of the consequences has been the appearance of Global Manufacturing Virtual Networks (GMVNs), which include all kinds of enterprises and production centres and establish a new type of horizontal collaboration and relations between independent companies and even competitors who establish occasional collaborations on projects they could not take on individually. This paper analyses the causes behind the formation of such networks, their strategy, structure, dynamics and evolution, taking into account areas such as strategic intercompany alliances, synchronization of their value and supply chains, their information systems, the cultural aspects of the organizations in question and, finally, their convergence with another of the more relevant future trends in production: mass customization. The proposed model shall be applied to the aeronautical industry which is one of the industries which has developed the GMVN concept. The case study of the engine manufacturer Rolls Royce will provide a better understanding of the evolution of its strategic positioning, as well as the dynamic and fluent nature of its virtual relations. This will demonstrate its effectiveness by clarifying and putting these organizations in perspective and analyzing their evolution over the next few years.
\end{abstract}

\section{Introduction}

Today, the concept of plant or production centre is becoming increasingly more ambiguous. In many industries, there is growing collaboration between production centres and manufacturing networks that seek to respond to market demands more efficiently and obtain competitive advantages in an increasingly globalized environment. In some industries, such as the aeronautical industry, the electronics industry or the car industry, there is mention of Global Manufacturing Virtual Networks (GMVNs) based on a new manufacturing architecture model with a high development potential to satisfy an increasingly demanding and fragmented market. In short, these networks represent a compendium of the new tendencies within the production organization, such as global manufacture, strategic alliances, flexible production and mass customization.

The purpose of this study is to analyze how GMVNs appear in the market and evolve in the future by considering their main characteristics that will determine their strategy positioning through a period of time. Some special tools will be proposed to study this strategic decision that will determine the starting point of the GMVN building process. Subsequently, another network features and their potential evolution will be considered to achieve a better understanding of how and why these organizations work. For achieving that purpose a case study about the engine manufacturer Rolls-Royce will be developed by analyzing already established and known facts on a new perspective that permits to have a comprehensive understanding about GMVNs. The initial descriptive approach about GMVNs, mostly developed in Section 5 , based on the new perspective given by the conceptual models defined in Figs. 2 and 5 will permit to gain a broad understanding about how these organizations work. Finally subSection 5.3 and Section 6 give some prescriptive propositions about how GMVNs should work and evolve in the future to be efficient.

The environment in which enterprises currently work with increasingly globalized markets, company consolidation and strategic alliances is forcing companies to find new forms of collaboration to improve the integration and synchronisation of the various functions and stages of their product value chain (Zhao et al., 2001). Global manufacturing virtual networks allow companies to focus on their core competences, maintaining their participation in the design and manufacture of complex integrated systems. These networks can be considered as extended manufacturing systems where various companies can co-operate on a specific project whose result is the manufacture of a product or the provision of a service and where each company is expert in one or more of the areas that give the product its value (Elmuti and Kathawala, 2001). 


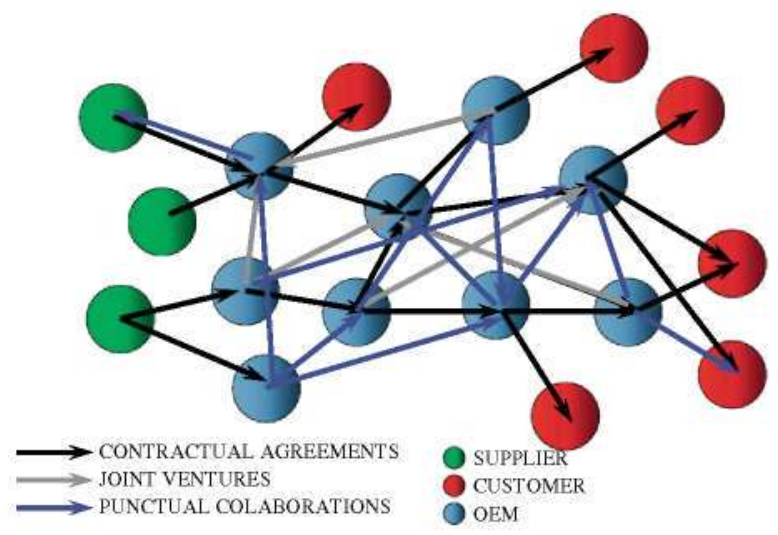

Fig. 1. Structure of global manufacturing virtual networks.

Although there are hardly any theoretical models or studies on how these networks function, they are known to develop on a large scale and involve a complex number of participants that include enterprises, organizations and institutions covering several countries or even continents. The implications in the various manufacturing fields are manifold and knowing how they are structured, how they coordinate and plan their needs and implement their supply chain management, what their specific competences are and how the different members of the network communicate shall be some of the features this paper seeks to clarify. In addition, market demands for increasingly customized products and services lead to the implementation of new manufacturing techniques such as mass customization, where the complexity of the implementation, the information flow or the planning of resources complicate the management of this type of network even further. Fig. 1 gives a simple example of the structure of this type of network, together with the relations between the nodes.

\section{Literature review and theoretical background}

First studies about multi-plant organizations started by the early 1980 s. These works were principally based on location criteria. When a network structure was implanted each factory was considered as an independent centre ignoring the network structure (Schmenner, 1982) and potential synergies. During these years, even though globalization of markets started to rise, operations and production organization studies were only concerned about independent manufacturing centres.

In the late 1980 s and early 1990 s, due to the intense growing demand of global markets, many companies seriously considered the benefits of manufacturing networks interconnected. A number of scholars have approached network manufacturing research from different perspectives: Shi et al. (2003, 2005), Hanna (2007), Rudberg and Olhager (2003, 2008) and Demeter (2003) assessed these organizations from a strategic approach. Shi and Gregory (1998) analyzed the interdependence of manufacturing centres where all matrix connexions where considered. Khurana and Talbot (1999) studied how each factory could influence one to each other in a network structure. Ferdows (1997), Sturgeon $(2000,2002)$, Kulmala et al. (2002), Williams et al. (2001) and Colotla (2002) focused on structural issues. Yusuf et al. (2004), Arshinder et al. (2008) and D'Amours et al. (1999) analyzed their communication systems and Sturgeon (2002) surveyed the cultural aspects of these networks.

First manufacturing networks were constituted by a number factories dispersed geographically to obtain certain competitive advantages like access to low production costs, qualified labour and proximity to strategic markets (Ferdows, 1997 and Bhutta et al, 2003). Anyhow, all these manufacturing centres were mostly owned by one or very few companies. Thus the "virtualization" degree of the network was very limited. It is understood that the virtual component of the network is related to the intensity of the collaborations with companies external to the organization itself (Shi and Gregory, 2003). This is the case of DEC where Arntzen et al. (1995) analyzed how digital equipment corporation redesigned its network including the relocation of some of its nodes at a corporate level. Other relevant studies about manufacturing networks with low "virtualization" are Acer (Mathews and Snow, 1998), Procter \& Gamble (Camm et al., 1997) or Hewlett Packard (Lee and Billington, 1995).

Li et al. (2000) were the first to name global manufacturing virtual networks by proposing a strategic positioning model for these organizations based on three vectors: globalization, strategic alliances and value and supply chains. These networks are more complex structures formed by several companies and several production centres based on horizontal and vertical relations among independent companies or even competitors that establish punctual collaborations in projects they could not afford individually (Tuma 1998). In some industries such as aeronautics (Shi et al., 2005), electronics (Shi and Gregory, 2003) or the automotive industry (Sturgeon and Florida, 2000), global manufacturing virtual networks (GMVN) has become a growing phenomenon with a high potential for development in order to satisfy an ever more demanding and fragmented market (Shi and Gregory, 2003).

During last years, many authors have studied this new phenomenon of virtual organizations (eg.: Corvello and Migliarese, 2007; Manthou et al., 2004; Offodile and Abdel-Malek, 2002). Other relevant works about GMVNs were made by Johansen and Comstock (2005) who analyzed the production strategy change of the aeronautical manufacturer SAAB AERO to join the Airbus manufacturing network. Meixell et al. (2004) studied the convergence of these organizations with mass customization systems and Williams et al. (2001) surveyed the relations among supply chain members and offset strategies in the global aerospace sector.

During last years, many scholars have approached GMVNs phenomenon from different perspectives to achieve a detailed understanding of some network features like their structure, information systems or alliance models. Anyhow, some research fields need further development like collaborative strategies among network actors or cross-cultural attributes at network level. Future works should also deep into a comprehensive understanding of all GMVNs features and their interdependence. By understanding the main building blocks of these organizations and their management processes, it will be possible to formulate a strategy and design process for developing effective GMVNs in the future.

\section{Research design and methodology}

This paper is a part of a research work about GMVNs modeling. The starting point has been a survey during six years (1999-2005) to the most relevant engine manufacturing plants within the aeronautical industry in Europe. Data was collected through a number of visits to the most relevant facilities in Europe: Volvo Aero (Trollhättan, Sweden), SNECMA (Evry-Corbeil, France), MTU (Munich and Hannover, Germany), GE Aero (Caledonian, UK; Eskiseir, Turkey), ITP (Zamudio and Ajalvir, Spain), IAI (Israel) and Rolls-Royce (Hillington, UK; and Oberursel, Germany). Methodology used based on the research terminology of Yin (1994) has been exploratory and descriptive based on a case study with 
qualitative approach and the data collection method has been open interviews to relevant executives of these facilities, and documentation. Exploratory studies are appropriate when the research problem is difficult to delimit, the problem is not well known and the available knowledge is not absolute (Yin, 1994). Additional methods of data collection were used to triangulate the data obtained from the interviews like company documentation (annual reports, key financial data, shareholders information, catalogues and company web page), professional market reviews, specialized internet sites and literature review.

The purpose of this study is to gain a better understanding of how and why these organizations work. For achieving that purpose a case study about the engine manufacturer Rolls-Royce will be developed by analyzing already established and known facts on a new perspective that permits to have a comprehensive understanding about GMVNs. Thus a qualitative approach will permit to study this phenomenon from the inside and gain a deeper understanding rather than a quantitative approach more convenient when clearly stated hypothesis can be tested on welldefined narrow studies (Yin, 1994). A qualitative case study approach has a distinct advantage when how or why questions is being asked about a contemporary set of events over which the investigator has little or no control. Yin (1994) also affirms that since case studies rely on analytical generalizations, which should try to generalize findings to theory, external validity should be high. However since this work only involves one case study, the ability to generalize is rather low. Thus more extensive case studies to other similar companies or to another industrial sectors where GMVNs are starting to grow to a great extent (e.g.: automotive or electronics industries) might stress the findings of this work in the future.

\section{Development of the conceptual framework}

To study this new phenomenon of collaboration between production centres and understand the nature of global manufacturing virtual networks in more detail, a conceptual framework is proposed in accordance with the diagram shown in Fig. 2, based on Ayers's customer-centric model (2002) and the proposal put forward by Johansen and Comstock (2005); the said framework is to be used as a platform for analyzing this type of network. This diagram enables the sequential analysis of all the factors that affect the design of a global manufacturing virtual network, such as its strategy, structure, communication systems and network culture.

The strategy of GMVNs is one of the network features to bear in mind. The manufacture of some aeronautical motors involves the participation of the great majority of manufacturers (competitors) on the market, as in the case of the GP 7200 which powers the new Airbus 380 whose manufacture is being done by an alliance between General Electric and Pratt \& Whitney with collaborations from MTU Aero Engines, Snecma, and Tech Space Aero. This paradox is permitted by the OEMs because the benefits obtained through this collaboration are much greater than the inherent risks of collaborative manufacturing. The close collaboration between competitor OEMs in the same sector is very frequent in GMVNs and, therefore, one can consider the validity of the classical two-dimensional Porterian model. Strategy in GMVNs follows different patterns and, for a better understanding of this phenomenon, a three-dimensional model for strategic positioning will be developed in this work.

The network structure feature includes the performance of its main actors (the nodes of the network), as well as the type of relations and collaborations that are established among its members. These collaborations can be long-term based strategic alliances (e.g.: CFM 56 aero engine manufactured by a $50-50$ joint venture between GE and SNECMA) or punctual collaborations with high virtual relations. The nature of its nodes (network actors) and their relations must not just be regarded as something static and rigid but instead as a system undergoing a continual process of change with, in some cases, diffuse and variable structure.

The third relevant network feature of GMVNs is the communication system which the network has. This feature would include all IT and communications tools the network needs to operate. Many authors have studied this field such as the work by Li et al. (2004) on manufacturing grids or Jiao et al. (2006) on collaborative manufacturing. These studies analyze how to coordinate the utilization of design and manufacturing resources that are heterogeneous, independent and distributed throughout the network. In the new development of the model 787 Boeing, a new concept of virtual design and manufacture has been implemented known as Global Collaborative Environment formed by a platform on the Internet which links up all the participants, internal and external, in the project, independently of their location, and permits them to jointly design and virtually simulate not just the functioning of the parts independently but

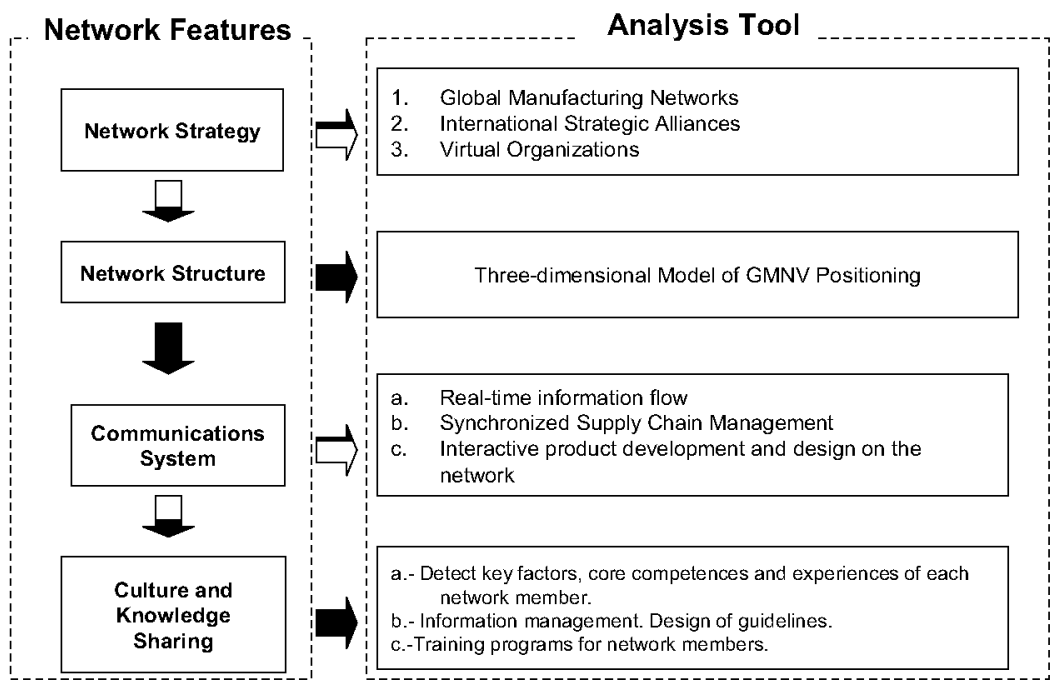

Fig. 2. Evaluation model of global manufacturing virtual networks. 
also the entire process of structural subunits of the plane. A final relevant aspect of GMVNs is their culture. Analyzing how to overcome the fear inherent to collaborating with companies outside of one's organization, in some cases competitors are one of the challenges of GMVNs. There are some cultural mechanisms that may highly influence the efficiency of these organizations like cultural similarity among different network actors, the importance of trust as a mechanism to avoid opportunistic behavior, the transfer of tacit knowledge and the social embeddedness (Granovetter, 1985). These cultural mechanisms may act previously to the start of the collaboration (ex-ante mechanisms) or once the relation is established (ex-post mechanisms) in a mutual reinforcing process that may infer a drastic reduction on transaction costs within GMVNs.

The mutual dependence and influence of these four network features is something that should be taken into consideration. The degree of "virtualization" of a GMVN will be inversely proportional to the intensity of formal and informal information flows and will influence on the type and efficiency of communications systems in the network. The strategy chosen (e.g.: more international alliances with external companies to have access to new markets, diversify financial risk or access to new technologies) will influence on the network structure (e.g.: increase of virtual relations), communications systems (e.g.: supply chain synchronization or harmonization, Rudberg and Olhager, 2003) and cultural aspects of the GMVNs. This paper will deep on the strategy network feature by analyzing a practical case in the aeronautical industry, i.e. the global network of the engine manufacturer Rolls-Royce (RR).

\section{Global manufacturing virtual networks in the aeronautical industry. A practical case of Rolls-Royce}

Aeronautical industry appeared at the beginning of the 20th century and, in a short period of time, has adopted various production strategies and organization methods ranging from the craft processes of its beginnings to the global manufacturing virtual networks that constitute the way in which commercial aircraft are manufactured today, including the mass production systems that were implemented during the second world war, technological innovations and the future mass customization systems that will constitute the trend of this century (Fig. 3 ). It is a compendium of the ideal way in which production has evolved and can be used to apply the conceptual model described above.

Rolls-Royce is currently one of the world leaders in the manufacture of engines for the civil and military aeronautical industry. The structure of the Rolls-Royce manufacturing centre network in the world is organized in such a way that each centre specializes in one or more engine components, which enables greater technical specialization and larger economies of scale. As a result, today, unlike the organization it had in the 1960s and 70s, no single centre is capable of manufacturing an entire engine.

To see how the network is structured, it must be understood that for each engine model or project, there will be a different supply chain on the network itself in accordance with a set of basic premises. Each manufacturing network will use the internal and external resources it requires, such as research centres, technology or component suppliers, own manufacturing centres, as well as horizontal collaborations with companies that manufacture engines.

Fig. 4 shows a simplified diagram of an aircraft engine to give an example of how its components might be manufactured at the various manufacturing centres on the network, the result of the collaboration between GE and RR on a new engine.

\subsection{Global manufacturing virtual network strategy and structure}

At present, companies need to focus strategically on their main competences to offer greater value added in the supply chain. Accordingly, a growing trend today is the subcontracting of the manufacturing process to external collaborators or suppliers. The traditional relations between vertically integrated manufacturers, component suppliers and distributors are currently under reconsideration and being compared with horizontal business collaborations between OEMs (original equipment manufacturers), highly specialized technology companies, component suppliers and distributors that form dynamically changing collaboration networks depending on each product, client and moment in time. In these global manufacturing virtual networks, the main company does not need to maintain internal manufacturing resources to cope with unpredictable variations in demand (Li et al., 2000). Rather they are based on relations with the various components of a virtual network that enables the company to design a specific supply chain in accordance with each client or specific contract. Therefore, this type of network is

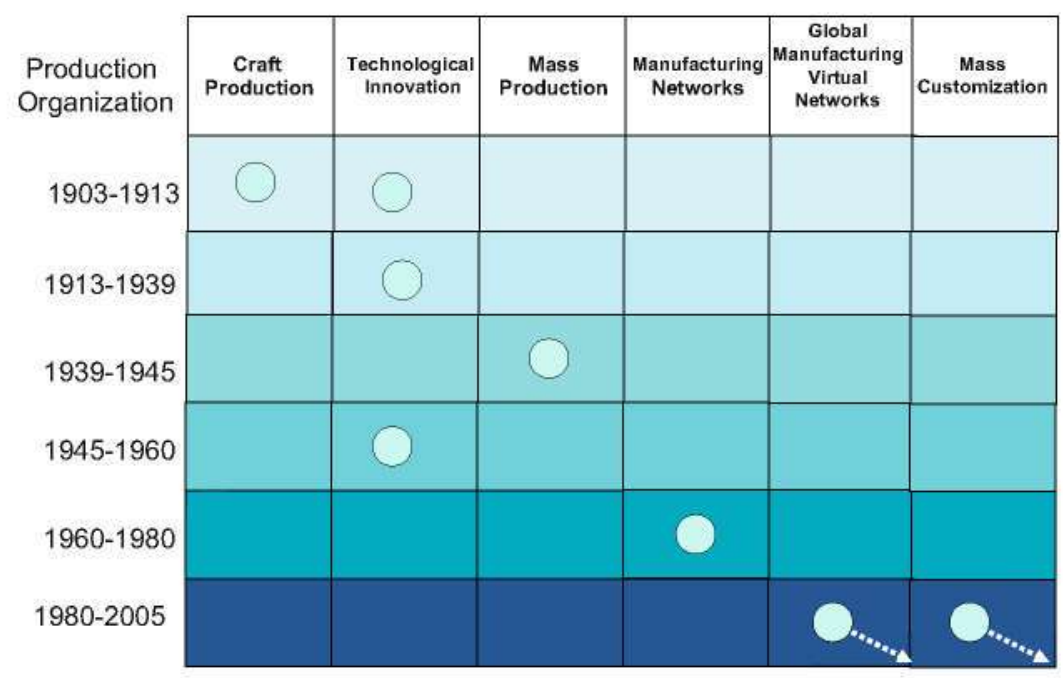

Fig. 3. Production organization evolution within the aeronautical industry. 


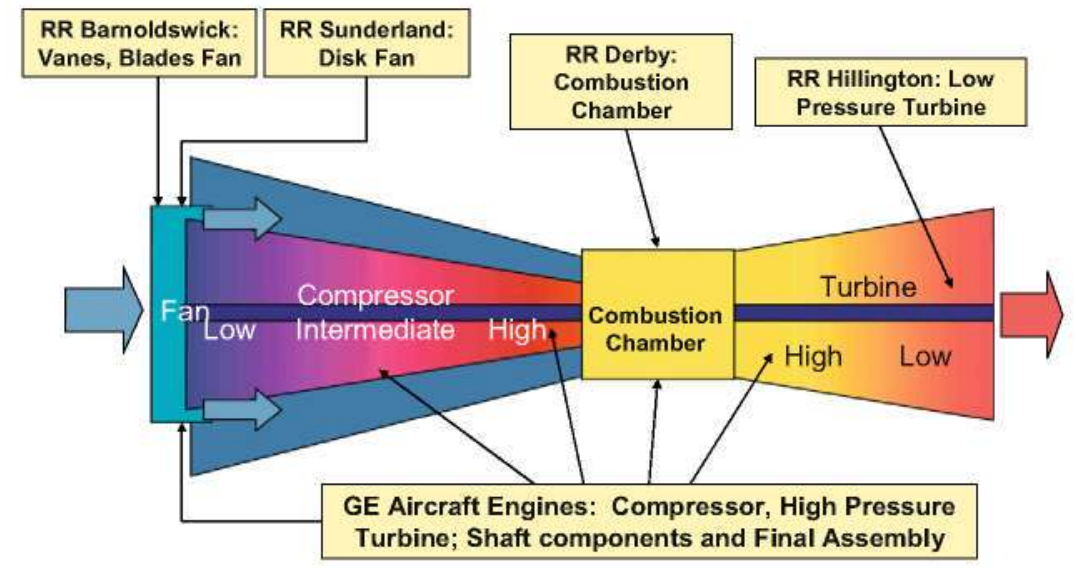

Fig. 4. Example of a new engine manufacturing diagram in a GE-RR collaboration.

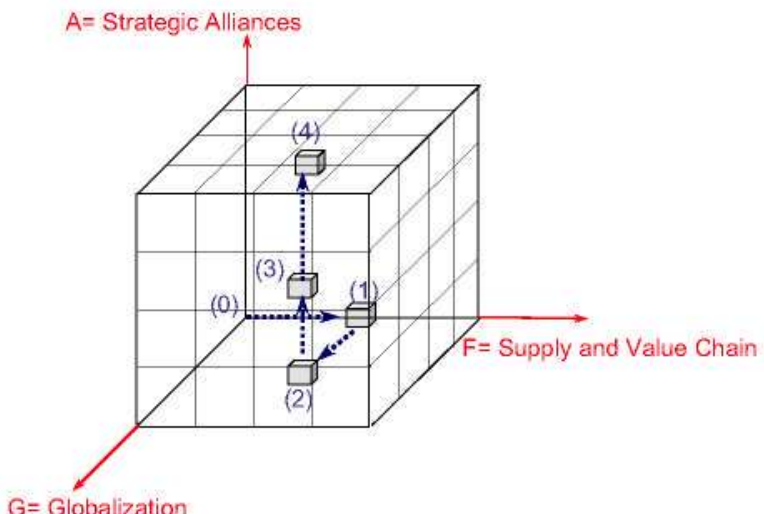

Fig. 5. Strategic positioning of Rolls-Royce global manufacturing virtual network.

not based on the possession of certain resources that condition what can be produced, when, and how much, but rather on managing and sharing the network resources.

Today, the formation of GMVNs follows four strategic focuses: (1) operative excellence; (2) access to new markets - geographical, product, client segments and compensation strategies (Williams et al., 2001); (3) diversification of financial risks; and (4) access to new technologies.

One of the most significant trends in the changes occurring to today's manufacturing systems, especially in the aforementioned industrial sectors, is the substitution of vertical integrations or relations with horizontal relations between competitors or enterprises specialising in very specific technologies to allow companies to focus on their main competences (Colotla, 2002). Paradoxically, this type of collaboration, which leads to a global manufacturing virtual network, will allow them to provide their clients with highly flexible global solutions depending on market demands at any given time.

When designing a global manufacturing virtual network, consideration must be given to the strategy of the preferred manufacturing system. Accordingly, four basic factors are taken into account:

(1) Internationalization of the manufacturing process: the manufacturing process is no longer considered as one single production centre, but rather has to include expansion or dispersion plants in accordance with the company's current strategy.
(2) Supply and value chain: the various tasks involved in the manufacturing systems and carried out on the network must be defined throughout the product value chain, and both the stage of the chain during which the tasks are to be carried out and the party by whom they are to be controlled must be specified. In addition, this comprehensive view of the process will enable optimization through the selection of internal and external activities, collaborators and the types of controls established, slightly increasing process efficiency and obtaining more competitive advantages.

(3) Strategic alliances: a very broad range of possible forms of intercompany collaboration must be assessed, ranging from specific collaborations on certain projects to long-term joint ventures or strategic alliances.

(4) Integration process: the three factors described above cannot be considered separately, but rather as part of an integrated manufacturing system in line with a preferred strategy.

\subsection{Global manufacturing virtual network positioning of Rolls-Royce}

These three dimensions, on which the design of the virtual network manufacturing system will be based, are described in Fig. 5 (Shi et al., 2005) and their result will define the positioning of the global manufacturing virtual network in which Rolls-Royce has changed its production strategy over time.

$\mathrm{G}$ represents the degree of globalization or internationalization of the manufacturing system.

F represents the creation of value obtained from the integration of the various supply and value chains formed by the companies that take part in the network, determined by the synergies and value added resulting from the integration of the various production centres.

A represents the level of collaboration between the various companies that define the strategic alliances on the network. Strong strategic alliances will allow for closer, long-term collaborations whereas more occasional collaborations will furnish the network with greater flexibility.

The vector resulting from the three-dimensional variables provides a qualitative idea of the type of global manufacturing virtual network in question.

It is interesting to note that in the design of traditional manufacturing systems, the dimensions of internationalization $(G)$ or collaboration (A) are rarely given consideration and only the impact of the manufacturing process on the product value 
chain (F) is considered. The current studies carried out on manufacturing systems are usually limited to two dimensions: on the one hand, the companies that consider only the $\mathrm{G} \times \mathrm{F}$ plane, where consideration is given to internationalization and the product supply and value chains, but collaboration with external companies is ignored; and, on the other, the $G \times A$ plane, which studies the internationalization of production processes and strategic alliances without considering the value added that could be obtained through the integration of the corresponding value chains. The overall view of the three dimensions has been the subject of little study and, therefore, the true potential of global manufacturing virtual networks has not been analyzed.

Point (0) of Fig. 5 refers to Rolls-Royce's beginnings in 1953, when it started up its aircraft engine manufacturing activity with a model called Dart, manufactured entirely at one of its plants in the United Kingdom. Point (1) shows the decentralization of the manufacturing processes begun by RR after it was privatized in 1987. Point (2) indicates the company's internationalization after privatization; however, this process did not include any significant collaboration with other companies. Point (3) shows the current situation, which presents a highly globalized company with manufacturing centres distributed all over the world (each centre specialising in one or more engine subunits), integrated supply chains that add value and a high level of participation in international projects in collaboration with other companies to form an authentic virtual network.

Point (4) in Fig. 5 indicates the company's trend for the coming years, where external collaboration will be used more and more to the point where the company's own resources shall be decapitalized. The future of this type of network is not based on the internal maintenance of manufacturing resources to satisfy unpredictable variations in demand. Rather it is based on relations with the various components of a virtual network that allow the company to design a specific supply chain in accordance with each engine type or client. Therefore, this type of network is not based on the possession of certain own resources that condition what can be produced, when it can be produced and how much, but rather on managing and sharing the network resources.

Fig. 6 is based on the three-dimensional figure shown before and can also be used as a very interesting analysis tool (Shi et al.,
2005). It shows the range of possibilities for positioning a network between virtual organizations (VO) and international strategic alliances (ISA). Global manufacturing virtual networks can be positioned within this spectrum in accordance with their strategy and based on the flexibility afforded by virtual organizations to attract new business opportunities or enter new markets and the ability of strategic alliances to increase levels of capacity and improve relations in the long term. This positioning should not be considered as an immovable, hierarchical concept, but rather as a dynamic positioning that can be modified in time in accordance with the market situation as it changes.

Fig. 7 shows some of the engines and projects in which RollsRoyce takes part. The more stable projects appear at the top of the table, with more lasting relations between the members of the network in the form of strategic alliances, as is the case with the Trent engine family and the company's long-term relations with enterprises such as ITP. For projects at earlier stages, where the risk is higher, such as the F136 engine, specific horizontal relations are established. Between the two, there are projects

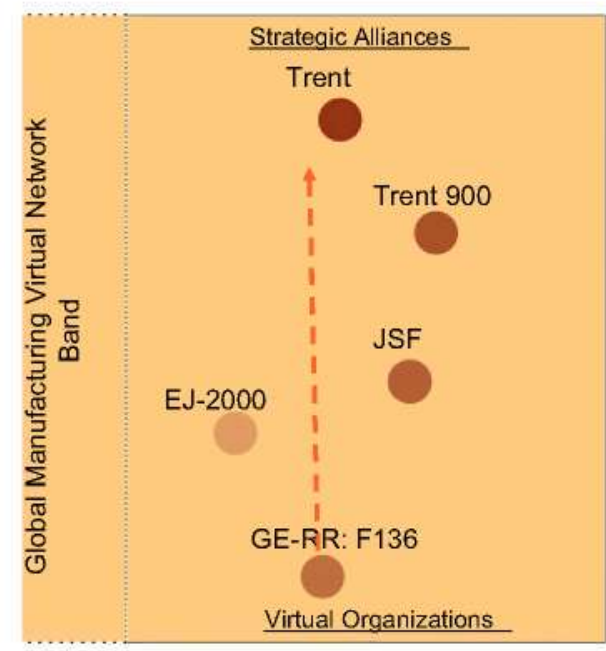

Fig. 7. GMVN positioning depending on each project.

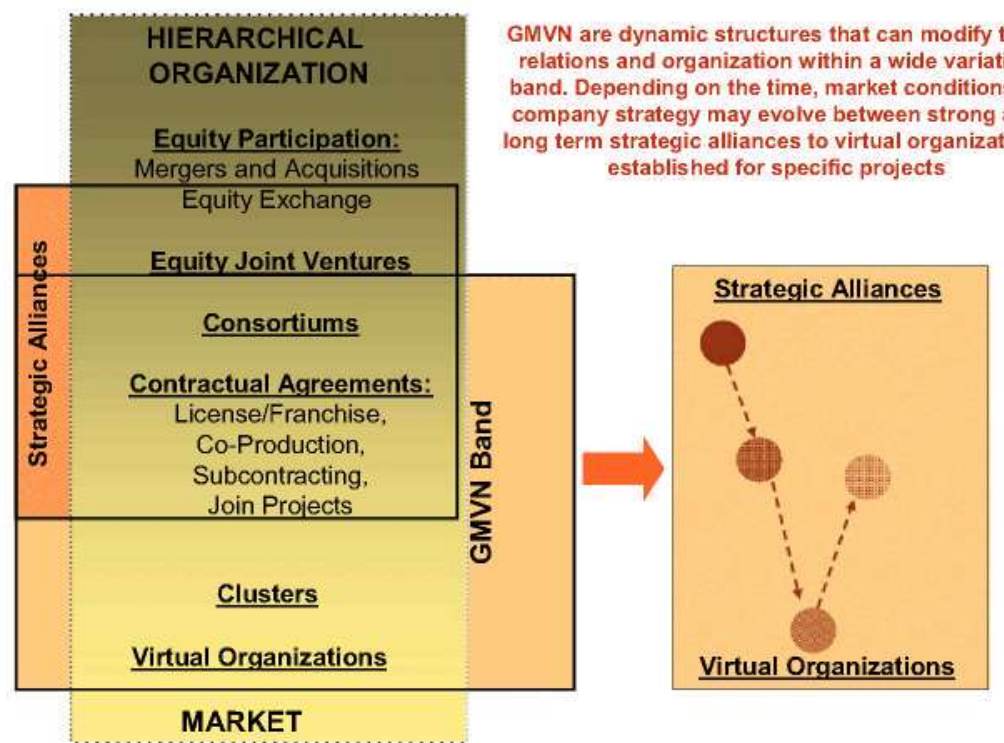

Fig. 6. GMVN Band. 
such as the new Trent 900 engine for the Airbus A380, based on collaboration with external companies, albeit true that the project is still clearly led by Rolls-Royce.

First Trent engine program was announced in 1988 to compete against General Electric and Pratt \& Whitney engines that, at that time, dominated the large engine civil market. Due to the enormous development costs required to bring a new engine to market, RR had to choose between establishing external collaborations to diversify the financial risk or to develop a family of engines based on an existing common core. At that time, RR considered that its RB211 engine could be the perfect core engine for the new family as its high pressure, intermediate-pressure and low-pressure systems could be individually scaled and customized to the requirements of the new engine. Thus RR chose a strategy of low "virtualization" by developing and manufacturing this new engine internally. Trent 500, 600 and 700 were the first models of this family based on the core engine of the former RB211 model and they were mostly manufactured within RR centres of Hillington, Ansty, Barnoldswick, Bristol and Sunderland. But not all Trent engines have such a low degree of external collaborations. The Trent 900 designed to power the new Airbus 380 is the most "virtual" engine within the Trent family. Due to the financial risk of this project and the high technological complexity, RR decided to collaborate with seven partners: Volvo Aero (intermediate compressor case), Marubeni Corporation (engine components), ITP (low-pressure turbine), Hamilton Sundstrand (electronic engine controls), Avio S.p.A. (gearbox module), Goodrich Corporation (fan casings and sensors) and Honeywell (pneumatic systems). Even though its level of "virtualization" cannot be considered high since this project is completely led by RR and some of this partners are RR joint ventures (e.g.: ITP).

In the opposite side of Fig. 7 it is placed the F136 engine manufactured by General Electric and Rolls-Royce exclusively for the F-35 Lightning II. This engine will be manufactured by GE (60\%) in its manufacturing plant of Cincinnati and by RR (40\%) in its plants of Bristol and Indianapolis. RR is responsible for the front fan, combustor and turbine while GE is in charge to develop the high pressure compressor, the augmentor and some turbine components. Due to the high complexity of its technical specifications, the extraordinary financial requirements $(\$ 2.4$ billion contract) and the continuous political inferences, the "virtualization" of this engine is of maximum level. Actually this project is involved in the system development and demonstration (SDD) phase (Phase IV) based on a contract award in 2005 consisting of 12,000 testing hours, first flight tests expected for 2009 and first production engine delivery in 2011. Once all development phases are accomplished, engine tests are successfully qualified and market demand is guaranteed the project could move to less virtual positions by establishing, for instance, a longterm joint venture between both partners.

The EJ200 engine, included in Fig. 7, is a typical example of collaborative manufacturing within the aeronautical industry. It is a military turbofan engine to power Eurofighter Typhoon and manufactured by the consortium Eurojet Turbo $\mathrm{GmbH}$ formed by Rolls-Royce (34,5\%), Avio (19,5\%), ITP (16\%) and MTU (30\%). Every partner is responsible for the development and production of some components according to its expertise and competence in every particular field. For example RR is in charge of the combustion system, high pressure turbine and engine health monitoring system. Even though its degree of "virtualization" is high due to the level of external collaborations, it does not reach the level of F136 engine since this engine is manufactured by a joint venture formed in 1986 . The final assembly stage follows an offset strategy (Williams et al. 2001) very typical within the military engine aerospace industry where the work share of each of the partner reflects the number of engines ordered by each country.

\subsection{Rolls-Royce GMVN case Discussion}

The aim of RR network is to satisfy a number of requirements that previously limited its expansion policy, such as the possibility of incorporating technological innovations into its engines, reducing financial risks with regard to new engine projects, reducing its own manufacturing resources by subcontracting subunits to collaborating companies, with each centre specialising in one type of engine technology, and achieving economies of scale by the production process of each manufacturing centre on the network specialising in one or more engine components or subunits. In addition, by reducing its manufacturing resources, Rolls-Royce has become more flexible with regard to reacting to changes in market demand, maintaining the main competences of its organization, which, within the scope of the manufacturing process, include the design and development of the engines and the assembly and final testing stages.

Rolls-Royce's strategy on the network is to change the supply chain in accordance with the project or engine type in question. In the case of its Trent engine family, there is very little horizontal collaboration, since it has a highly consolidated position on the market and has been manufactured for many years. The manufacturing process is carried out at Rolls-Royce manufacturing centres or companies in which the company has significant holdings in the share capital. Each centre makes one or more engine components that are finally assembled and tested at its facilities in Derby in the United Kingdom. However, in the case of the new F136 engine, the supply chain is based on horizontal collaboration with manufacturers, in some cases, direct competitors (e.g. GE), to form an authentic virtual network whose external participants work together on the specific manufacture of the engine. The benefits of this type of collaboration are beyond question since they allow an approach to high-financial-risk projects, resulting in greater technical specialization of the components and a highly flexible production.

The three-dimensional model of Fig. 5 applied to the production strategy of Rolls-Royce over time has provided a qualitative idea of the evolution of its manufacturing strategy since the beginning and to estimate the trend for the coming years. This tool may permit to compare the strategic positioning of RR with other engine manufacturers or to estimate the better strategy for the future. Figs. 6 and 7 have provided a good idea of the nature and dynamics of external collaborations of RR manufacturing network as well as the flexibility that can be adopted depending on each engine or project.

The development of virtual relations by companies within GMVNs is a good strategy when they want to enter in new projects and thus diversify financial risks or have access to new technologies. Anyhow, the more these projects consolidate (e.g. stable market share or technology maturity), the less "virtualization" degree they should have to avoid or minimize some inherent risks of these kind of collaborations like technology transfer, role cannibalization or loss of manufacturing process control. Virtual relations are very useful, as well, to allow medium-small size companies to enter in GMVNs and participate in projects or products they could never afford to accomplish independently.

The RR case studied has mostly analyzed the strategy network feature of RR included in Fig. 2. The different strategy positions of RR over time will have a strong impact on other network features like its structure, its information systems and its culture that could be extensively studied in future research works to complete the findings of this work. 


\section{Future evolution of global manufacturing virtual networks in the aeronautical industry: mass customization and "network virtualization"}

The challenge now is to forecast what the next step will be, how the manufacturing systems will evolve in the coming years with regard to these global manufacturing virtual networks and what the determining factors behind the development of this industry will be. Basically, there are two clear trends in the production strategies of these networks in the aeronautical industry: (1) the convergence between this type of network and mass customization systems and (2) a greater "virtualization" of the networks leading to the decapitalization of a large part of the companies' manufacturing resources in favour of a greater specialization in one or more aircraft engine components.

\subsection{Mass customization in the GMVN of the aeronautical industry.}

According to McClellan (2003), Mass customization is a growing market demand that will require manufacturing networks to implement production systems that address the said demand for customized services and products at prices similar to those that are mass produced. Meixell et al. (2004) and Kamrani (2004) have also mentioned the importance of mass customization in the context of global manufacturing networks, highlighting the importance and difficulty of supply chain management in these environments.

Although it is not at all simple to consider all the possibilities of this convergence, it is possible to estimate the feasibility of the process and the profits that would be obtained. Global manufacturing virtual networks are based on a dispersed manufacturing process which, in most cases, implies the manufacturing of the product by modules or independent subunits or in completely separate stages of the product chain. As a result, the application of mass customization techniques, such as the modularization of products (Ulrich, 1995) or the customization during the latter stages of production is possible.

According to Bugos (2001), customization in this industry began with the introduction in 1970 of the Boeing 747. When this plane was supplied to the airlines of each country, it was discovered that each one had slightly different requirements, which forced Boeing, McDonnell Douglas and Airbus to implement new mass customization methods through the application of small final adaptations in their mass production processes, such as that implemented by Boeing in its wing production process. However, mass customization will be implemented on a large scale in the aeronautical industry as a natural process resulting from global manufacturing virtual networks and the varied requirements of an increasingly demanding market that seeks differentiation through customized aircraft that satisfy the particularities of clients or their strategic positioning on the market without increase in the price of the aircraft in question.

Within global manufacturing virtual networks, mass customization becomes a feasible model that was almost unthinkable in the past. The dispersion of the manufacturing process requires greater specialization in one or more specific components in comparison with the all-round solutions that were supplied previously. As a result, in this context, mass customization systems will be based on two fundamental trends: product modularity and superficial changes in the final stages of the value chain.

1. Product modularity: this will be one of the fundamental factors behind the effective implementation of mass customi- zation in the aeronautical industry. Product modularity refers to a design of the product that allows for the combination of different components or subunits in such a way that the client can choose from several options for each module. This feature can be implemented seamlessly in global manufacturing virtual networks, since the network involves the dispersion of manufacturing processes and, for the said processes to be efficient, some kind of product modularity is necessary so that its manufacture can be broken down into similar subunits for later assembly. The example of the manufacture of the Airbus A380 is a very good one, since each country taking part in the consortium is responsible for one of the structural subsystems of the aircraft (fuselage, wing, cabin or tail), which are manufactured separately with their own supplier networks, subunit suppliers and collaborators until the manufacture of the subsystem is completed and sent to Toulouse (France) for the final assembly stage. In other words, by dispersing the manufacturing processes over a network, the modularity of the product design is almost a fundamental requirement for the efficiency of the supply chains. Therefore, when implementing mass customization systems based on modular designs, half the work has already been done.

2. 'Superficial' changes: mass customization through changes in the final stages of the value chain will be one of the most effective techniques to be implemented in the aeronautical industry. Many manufacturers have uncovered the market's need for changes to the variety and specifications of the aircraft and at structural subsystem level (wings, engines, fuselage, cabin, etc.); however, at the same time, they have found that the existing processes can satisfy these changes in the demand. In other words, new market requirements focus on 'superficial' changes to the aircraft which, in most cases, do not require substantial changes to either the manufacturing networks or the internal production processes. The new Airbus A380 has already implemented this option by offering various internal configurations of the inhabitable area of the aircraft and, in the coming years, the concept will be applied to other subunits with no substantial effect on the manufacturing processes. The origin of this trend lies in the requirements of a market that is becoming more and more demanding and versatile and seeks to provide its clients with a differentiated service.

\section{2. "Virtualization" of the network in the aeronautical industry}

Another trend that will gain in significance in the production strategy of these networks in the aeronautical industry is the increase in virtual collaborations between external companies for specific projects. The benefits of this type of collaboration will mean that companies will not need to maintain internal manufacturing resources, since they will be able to use the network resources and assume greater flexibility to satisfy unpredictable variations to the demand. The manufacturing systems of the future will be based on relations with the various components of a virtual network that enable the company to design a specific supply chain in accordance with each engine type or client. In short, this type of network will not be based on the possession of specific resources, but on managing and sharing the resources available on the network.

Consequently, as with other manufacturing companies, RollsRoyce shall tend more towards specialization. Participation in every stage of an engine's value chain will no longer be profitable and companies will have to specialise in a number of main competences, such as design or a specific structural subunit 
(e.g. a compressor), leaving the responsibility for other structural subunits or specific technologies to other companies on the network.

\section{Conclusion}

Global manufacturing virtual networks are based on three basic vectors: the globalization of internal manufacturing processes; the supply and value chains of all the centres involved; and strategic alliances with companies outside the organization. Although this type of intercompany collaboration is becoming more and more common, especially in the aeronautical industry, the car industry and the electronics sector, there are currently no models that describe how the networks operate or how they should be managed or designed. Their future growth potential is huge, since, on the one hand, they are more efficient at meeting the requirements of a market that is becoming increasingly varied and variable in its search for customized solutions at very competitive prices and, on the other, they allow manufacturers to reduce their financial risk by disinvesting heavy internal manufacturing resources, access new markets and seamlessly incorporate technological improvements to their products. In addition, the future evolution of this type of network will be based on a greater "virtualization" of the network and a convergence with mass customization systems.

The conceptual framework proposed in Fig. 2 permits a sequential analysis of all the factors that affect the design of a Global manufacturing virtual network, such as its strategy, structure, communication systems and network culture as well as to take into consideration the mutual dependence and influence of these four network features. The three-dimensional model of Fig. 5 applied to the production strategy of Rolls-Royce over time has provided a better understanding of the evolution of its strategic positioning since its beginning and has permitted to estimate its evolution for the next years. This powerful tool may permit to compare the strategic positioning of different companies and estimate the better strategy for the future. Figs. 6 and 7 have provided a good understanding about of the dynamic and fluent nature of the virtual relations in a GMVN as well as the flexibility that can be adopted depending on each engine or project. Anyhow, since this work has mostly been focused on the strategy network feature of $\mathrm{RR}$, future research works could extend the analysis to other network features like its structure, its communication systems or its culture that have evidently been affected by the different strategy positions of RR over time. This approach might stress and complete the findings of this work in the future.

\section{References}

Arntzen, B.C., Brown, G., Harrison, T.P., Trafton, L.L., 1995. Global supply chain management at Digital Equipment Corporation. Interfaces 25 (1), $69-93$.

Arshinder, Arun Kanda, A., Deshmukh, S.G., 2008. Supply chain coordination: perspectives, empirical studies and research directions. International Journal of Production Economics 115 (2008), 316-335.

Ayers J., 2002. CASA/SME blue book: supply chain management and the manufacturing enterprise wheel. Computer and Automated Systems Association of the Society of Manufacturing Engineers.

Bhutta, K.S., Huq, F., Frazier, G., Mohamed, Z., 2003. An integrated location, production, distribution and investment model for multinational corporation. International Journal of Production Economics 86/3, 201-216.

Bugos, Glenn, 2001. History of the Aerospace Industr. EH.Net Encyclopedia, edited by Robert Whaples. August 29, 2001.

Camm, J.D., Chormann, T.E., Dill, F.A., Evans, J.R., Sweeney, D.J., Wegryn, G.W., 1997. Blending OR/MS, judgment, and GIS: Restructuring P\&G's supplychain. Interfaces (1997) 27 (1), 128-142.
Colotla, I. 2002. Global Manufacturing Strategy Process: a Proposal for a Process Approach Integrating Factory and Network Perspective, In: Seventh Annual Cambridge International Manufacturing Symposium Proceedings, Cambridge, UK

Corvello, V., Migliarese, P., 2007. Virtual forms for the organization of production: a comparative analysis. International Journal of Production Economics 110 $(1-2), 5-15$.

D'Amours, S., Montreuil, B., Lefrançios, P., Soumis, F., 1999. Networked manufacturing: the impact of information sharing. International Journal of Production Economics 58, 63-79.

Demeter, K., 2003. Manufacturing strategy and competitiveness. International Journal of Production Economics 81-82 (2003), 205-213.

Elmuti, D., Kathawala, Y., 2001. An overview of strategic alliances. Management Decision 39, 205-217.

Ferdows, K., 1997. Making the most of foreign factories. Harvard Business Review 199775 (2), 73-88.

Granovetter, M., 1985. Economic action and social structure: the problem of embeddedness. The American Journal of Sociology 91 (3), 481-510.

Jiao, Jianxin (Roger), You, Xiao, Kumar, Arun, 2006. An agent-based framework for collaborative negotiation in the global manufacturing supply chain network. Robotics and Computer-Integrated Manufacturing 22 (2006), 239-255.

Johansen, K. Comstock, M., 2005. Coordination in collaborative manufacturing mega-networks: a case study. Journal of Engineering and Technology Management 22 (3), 226-244 Septiembre 2005.

Kamrani, A.K. (Ed.), 2004. Mass Customization: A Supply Chain Approach. Kluwer Academic Publisher pag.

Khurana A., Talbot B., 1999. Plant missions in global manufacturing networks: a resource-based view with evidence from the global color picture tube industry. Working Paper 99-0005. University of Michigan Business School, Ann Arbor, MI, 1999.

Kulmala, H.I., Paranko, J., Uusi-Rauva, E., 2002. The role of cost management in network relationships. International Journal of Production Economics 79, 33-43.

Hanna, V., 2007. Exploiting complementary competencies via inter-firm cooperation. International Journal of Technology Management 37 (3-4), 247-258.

Lee, H.L., Billington, C., 1995. The evolution of supply-chain management models and practices at Hewlett-Packard. Interfaces 25 (5), 42-63.

Li R., Yu T., Fang M., 2004. The reliability management of manufacturing grid, CIMS and Robot Center, Shanghai University, Shanghai, China, Flexible Automation and Intelligent Manufacturing, FAIM2004, Toronto, Canada.

Li, X., Y. Shi and M.J. Gregory, 2000. Global Manufacturing Virtual Network (GMVN) and Its Position in the Spectrum of Strategic Alliance, Operations Management: crossing borders and boundaries: the changing role of operations, In: Proceeding of the EurOMA Seventh International Annual Conference. Ghent, Belgium, pp. 330-337.

Manthou, V., Vlachopoulou, M., Folinas, D., 2004. Virtual e-Chain (VeC) model for supply chain collaboration. International Journal of Production Economics 87 $(3,18), 241-250$.

Mathews, J.A., Snow, C.S., 1998. A conversation with the Acer Group's Stan Shih on global strategy and management. Organizational Dynamics 27 (1), 65-74.

McClellan, M., 2003. Collaborative strategies: outside-the-box manufacturing. Optimize Magazine March 17.

Meixell, M.J., Wu, S.D., Kamrani, 2004. Collaborative Manufacturing for Mass Customization. Kluwer Academic Publisher, Boston, Massachusetts pag. 171-191.

Offodile, O.F., Abdel-Malek, L.L., 2002. The virtual manufacturing paradigm: The impact of IT/IS outsourcing on manufacturing strategy. International Journal of Production Economics 75, 147-159 January.

Rudberg, M., Olhager, J., 2003. Manufacturing networks and supply chains: and operations strategy perspective. OMEGA, octubre, 29-39 page.

Rudberg, M., Olhager, J., 2008. Global operations strategy: coordinating manufacturing networks. Omega 36, 91-106.

Schmenner, R.W., 1982. Multiplant manufacturing strategies among the Fortune 500. Journal of Operations Management 19822 (2), 77-86.

Shi, Y., Gregory, M., 1998. 1998; International manufacturing networks-to develop global competitive capabilities. Joumal of Operations Management $16(2,3), 195-214$

Shi, Y., Gregory, M.J., 2003. From original equipment manufacturers to total solution providers: an emergence of global manufacturing virtual network in electronics industry. International Journal of Service Technology and Management 4 (4-6), 331-346.

Shi Y., Fleet D., Gregory M., 2005. Global manufacturing virtual network and its position in manufacturing systems. In: Proceeding of the Seventh Annual International Manufacturing Symposium, Institute for Manufacturing, Department of Engineering, University of Cambridge.

Sturgeon, T. and R. Florida, 2000. Globalization and jobs in the automotive industry, final report to the Alfred P. Sloan Foundation, Center for Technology, Policy, and Industrial Development, Massachusetts Institute of Technology, Cambridge, MA.

Sturgeon, J., 2002. Modular production networks. A new American model of industrial organization; Industrial and Corporate Change 11 (3), 451-496.

Tuma, A., 1998. Configuration and coordination of virtual production networks. International Journal of Production Economics 5657, 641-648.

Ulrich, K., 1995. The role of product architecture in the manufacturing. Research Policy 24, 419-440. 
Williams T., Ellis B., Maull R., Gregory M., 2001. Offset Strategies in the Global Aerospace Sector. In: the Proceedings of the Sixth Research Symposium on International Manufacturing: Global Integration (ISBN 1-902546-23-7), Churchill College, Cambridge, 9-11 September 2001, pp. 153-166.

Yin, R.K., 1994. Case Study Research: Design and Methods. Thousand Oaks Sake Publications.
Yusuf, Y., Gunasekaran, A., Abthorpe, M.S., 2004. Enterprise information systems project implementation: a case study of ERP in Rolls-Royce. International Journal of Production Economics 87 (3), 251-266.

Zhao D., Shi Y., and Gregory M.,. 2001. Manufacturing Virtual Network System. In: the Proceedings of the Sixth Research Symposium on International Manufacturing: Global Integration, University of Cambridge, 2001 pp. 197-205. 